\title{
Kinetic Ballooning Instability As A Substorm Onset Mechanism
}

\author{
C. Z. Cheng \\ Princeton Plasma Physics Laboratory, Princeton University \\ Princeton, NJ 08543
}

\begin{abstract}
A new scenario of substorm onset and current disruption and the corresponding physical processes are presented based on the AMPTE/CCE spacecraft observation and a kinetic ballooning instability theory. During the growth phase of substorms the plasma $\beta$ is larger than unity $(20 \geq \beta \geq 1)$. Toward the end of late growth phase the plasma $\beta$ increases from 20 to $\geq 50$ in $\sim 3$ minutes and a low frequency instability with a wave period of $50-75 \mathrm{sec}$ is excited and grows exponentially to a large amplitude at the current disruption onset. At the onset, higher frequency instabilities are excited so that the plasma and electromagnetic field form a turbulent state. Plasma transport takes place to modify the ambient pressure profile so that the ambient magnetic field recovers from a tail-like geometry to a dipole-like geometry. A kinetic ballooning instability (KBI) theory is proposed to explain the low frequency instability (frequency and growth rate) and its observed high $\beta$ threshold $\left(\beta_{c} \geq 50\right)$. Based on the ideal MHD theory $\beta_{c}^{M H D} \simeq 1$ and the ballooning modes are predicted to be unstable during the growth phase, which is inconsistent with observation that no appreciable magnetic field fluctuation is observed. The enhancement of $\beta_{c}$ over $\beta_{c}^{M H D}$ is due to the kinetic effects of trapped electrons and finite ion Larmor radii which provide a large stabilizing effect by producing a large parallel electric field and hence a parallel current that greatly enhances the stabilizing effect of field line tension. As a result, $\beta_{c}$ is greatly increased over $\beta_{c}^{M H D}$ by a factor proportional to the ratio of the total electron density to the un-trapped electron density, $n_{e} / n_{e u}$, which is $\geq O\left(10^{2}\right)$ in the near-Earth plasma sheet. The wave-ion magnetic drift resonance effect produces a perturbed resonant ion velocity distribution centered at a duskward velocity roughly equal to the average ion magnetic drift velocity. This perturbed ion distribution explains the enhanced duskward ion flux during the explosive growth phase and can excite higher frequency instabilities (such as the cross-field current instability).
\end{abstract}




\section{Introduction}

A critical issue in the magnetospheric physics is the substorm process, in particular, the substorm onset and current disruption. Recently a new scenario of the substorm onset and current disruption has been presented [Cheng and Lui(1998)] based on the AMPTE/CCE spacecraft observations. In the late substorm growth phase the plasma $\beta$ at the substorm onset site in the near Earth plasma sheet region is typically $\sim 20$ at $\sim 10$ minutes prior to the current disruption onset. At $\sim 2$ minutes before the substorm onset $\beta$ increases to $\geq 50$ and the pressure becomes isotropic [Lui et al.(1992)]. Therefore, too much plasma energy is stored in the plasma sheet and the excess energy must be release via instabilities and associated plasma and magnetic field transport. We have found that a low frequency instability with a wave period of $\sim 50-75 \mathrm{sec}$ is excited and grows exponentially to a large amplitude with $\delta B / B \geq 0.5$ at the onset. The half wave period of the instability before the onset was previously called "explosive growth phase" [Ohtani et al.(1992), Ohtani et al.(1995)] which lasts $\sim 30$ sec with an enhanced duskward ion flux centered at $\sim 500 \mathrm{~km} / \mathrm{s}$ (below the ion thermal velocity of $\approx 1000 \mathrm{~km} / \mathrm{s})[$ Lui(1996)]. This enhanced cross-tail ion drift population is responsible for exciting higher frequency instabilities (with wave periods of $15 \mathrm{sec}, 10$, sec, 5 sec, etc.) which together with the low frequency instability last throughout the current disruption phase to form a strong turbulence for $\sim 4-5$ minutes. During the turbulent state anomalously fast plasma transport takes place to modify the average pressure profile so that the plasma sheet recovers to a lower energy state and the ambient magnetic field relaxes from a tail-like geometry to a dipole-like geometry.

Previously the ideal MHD ballooning instability has been proposed to explain the substorm current disruption and explosive growth phase [Roux et al.(1991), Liu(1997), Voronkov et al.(1997)]. The ballooning instability results from the release of free energy of nonuniform plasma pressure with a gradient in the same direction as the magnetic field curvature. Analogous to the expansion of a balloon due to higher inner air pressure around weak surface tension spot, the ballooning instability will relax higher plasma pressure and hence move the magnetic field lines across the large field curvature surface area toward the weaker pressure direction. Theories of ballooning modes based on the ideal MHD model would predict a purely growing instability when the plasma $\beta$ is above a low critical value $\beta_{c}^{M H D}$. In a dipole field or a more tail-like field $\beta_{c}^{M H D} \simeq 1$ with $L_{p}=1 \mathrm{R}_{\mathrm{E}}$. This is inconsistent with the AMPTE/CCE spacecraft observation that no appreciable magnetic field fluctuations were observed throughout most of the growth phase even when plasma $\beta$ values are above $\beta_{c}^{M H D}$ (typically $40 \geq \beta \geq 1$ ).

In this paper we present a kinetic theory of ballooning instability (KBI) which shows that kinetic effects of trapped particle dynamics, finite ion Larmor radii (FLR) 
and wave-particle resonances are important in determining the stability of ballooning modes. The kinetic theory of ballooning modes provides a proper understanding of two key physical processes of substorm current disruption and subsequent magnetic field dipolarization: (1) the excitation mechanism and the high plasma $\beta$ threshold $(\geq 50)$ of the low frequency instability that underlines the explosive growth phase; (2) the physical mechanism of the enhanced duskward ion flux that occurs only during the explosive growth phase and leads to excitation of higher frequency instabilities. In particular, we show that the kinetic effects of trapped electrons and finite ion Larmor radii produce a large parallel electric field and hence a parallel current that greatly enhances the

stabilizing effect of field line tension. As a result, the $\beta$ threshold $\left(\beta_{c}\right)$ for KBI is greatly increased over the ideal MHD ballooning instability threshold by $\geq O\left(10^{2}\right)$, which is consistent with the AMPTE/CCE observation of high $\beta_{c}\left(\beta_{c} \sim 50\right)$ for exciting a low frequency instability just before the substorm onset. The kinetic ballooning instability has a real frequency on the order of ion diamagnetic drift frequency which is associated with ion FLR effects. Moreover, the wave-ion magnetic drift resonance effect produces a perturbed resonant ion velocity distribution centered at a duskward velocity which roughly equals to the average ion magnetic drift velocity. This perturbed ion distribution explains the enhanced duskward ion flux during the explosive growth phase and can excite higher frequency instabilities (such as the cross-field current instability).

In the following we first describe the derivation of the eigenmode equations for kinetic ballooning instability based on the gyrokinetic formulation. We then present the physical process of the kinetic ballooning instability and its application to understand the critical substorm processes. Then, we discuss the difficulties in attempting to explain the substorm process by the magnetic reconnection based on the near-Earth neutral line model [Nagai and Kamide(1995)]. Finally, a summary is given.

\section{Gyrokinetic Formulation}

In order to properly address kinetic effects on the ballooning instability we will employ the gyrokinetic formulation to describe the particle dynamics. We shall consider collisionless plasmas with isotropic pressure. The particle velocity distribution function is assumed to have no appreciable bulk flow velocity. Quasi-static equilibria with isotropic pressure are determined by the system of equations in the rationalized EMU unit: $\mathbf{j} \times \mathbf{B}=\nabla P, \nabla \times \mathbf{B}=\mathbf{J}$, and $\nabla \cdot \mathbf{B}=0$. For three-dimensional magnetospheric equilibria the magnetic field can be expressed in a straight field line $(\psi, \alpha, \theta)$ flux coordinate as $\mathbf{B}=\nabla \psi \times \nabla \alpha$, where $\psi$ is the magnetic flux function, $\alpha=\phi-\delta(\psi, \alpha, \theta)$, $\theta$ is a generalized poloidal angle, $\phi$ is the azimuthal angle in the cylindrical $(R, \phi, Z)$ coordinate, and $\delta(\psi, \alpha, \theta)$ is periodic in both $\phi$ and $\theta$. The intersection of constant $\psi$ 
and $\alpha$ surfaces defines the magnetic field line. The flux coordinate system is in general not orthogonal, and $\nabla \psi \cdot \nabla \theta \neq 0, \nabla \psi \cdot \nabla \alpha \neq 0$, and $\nabla \alpha \cdot \nabla \theta \neq 0$. Within a magnetic surface the poloidal flux is $\Psi=\int d^{3} x \mathbf{b} \cdot \nabla \theta / 2 \pi=2 \pi \psi$. Note that $\alpha$ is a cyclic function with a period of $2 \pi$ for all constant $\psi$ surfaces.

We consider low frequency perturbations with $\omega<<\omega_{c i}$ and $k_{\perp} L>>k_{\|} L>1$, where $\omega$ is the wave frequency, $\omega_{c i}$ is the ion cyclotron frequency, $L$ is the equilibrium scale length, and $k_{\|, \perp}$ are the parallel and perpendicular wave numbers, respectively. Because the electron mass is much smaller than the ion mass and the temperatures of electrons and ions are of the same order, the electron thermal velocity is much larger than the ion thermal velocity. We consider electromagnetic perturbations with the orderings: $k_{\perp} \rho_{i} \sim O(1)$ and $v_{t h e}>\left(\omega / k_{\|}\right)>v_{t h i}[$ Cheng(1982b), Cheng(1982a)], where $\rho_{i}$ is the ion gyroradius. With these orderings the following kinetic effects must be considered: trapped electron dynamics, ion FLR effect and wave-particle resonance with $\omega-\omega_{d i}=0$, where $\omega_{d i}$ is the ion magnetic drift frequency. We shall obtain approximate solutions of the perturbed particle distributions based on the gyrokinetic formulation [Cheng et al.(1995)].

We assume a WKB eikonal representation for perturbed quantities, i.e., $\delta f(\vec{x}, \vec{v}, t)=$ $\delta f\left(s, \mathbf{k}_{\perp}, \vec{v}, t\right) \exp \left[i\left(\int d \vec{x}_{\perp} \cdot \mathbf{k}_{\perp}-\omega t\right)\right]$. Including full gyroradius effects the perturbed particle distribution function can be expressed in terms of the rationalized MKS unit as $\delta f=(q / M) \partial F / \partial \mathcal{E}\left[\left(\omega_{\star}^{T} / \omega\right) \Phi-\left(1-\omega_{\star}^{T} / \omega\right)\left(1-J_{0} e^{i \delta L}\right) \Phi\right]+g e^{i \delta L}$, where $M$ is the particle mass, $q$ is the particle charge, the guiding center particle equilibrium distribution is assumed to be $F=F(\mathcal{E}, \psi)$ so that the equilibrium pressure is a function of $\psi$ and $\mathcal{E}$ only, $\delta L=\mathbf{k} \times \mathbf{v} \cdot \mathbf{B} / \omega_{c} B, J_{l}$ is the $l$-th order Bessel function of the argument $k_{\perp} v_{\perp} / \omega_{c}, \omega_{c}=q B / M$ is the cyclotron frequency, $B$ is the magnetic field intensity, $\Phi$ is the perturbed electrostatic potential, and $g$ is the non-adiabatic part of the perturbed distribution function. Based on the WKB-ballooning formalism the gyrokinetic equation for $g$ in the low frequency $\left(\omega \ll \omega_{c}\right)$ limit is given by

$$
\begin{array}{r}
\left(\omega-\omega_{d}+i \mathbf{v}_{\|} \cdot \nabla_{\|}\right) g=-\frac{q}{M} \frac{\partial F}{\partial \mathcal{E}}\left(1-\frac{\omega_{\star}^{T}}{\omega}\right) \\
\times\left[\left(\omega_{d} \Phi-i \mathbf{v}_{\|} \cdot \nabla_{\|} \Psi\right) J_{0}+\frac{\omega v_{\perp}}{k_{\perp}} J_{1} \delta B_{\|}\right],
\end{array}
$$

where $\Psi$ is the parallel perturbed electric field potential with $\mathbf{E}_{\|}=-\nabla_{\|} \Psi, \delta B_{\|}$is the parallel perturbed magnetic field, $\omega_{\star}^{T}=\mathbf{B} \times \mathbf{k}_{\perp} \cdot \nabla F /\left(B \omega_{c} \partial F / \partial \mathcal{E}\right), \omega_{d}=\mathbf{k}_{\perp} \cdot \mathbf{v}_{d}$ is the magnetic drift frequency, $\mathbf{v}_{d}=\left(\mathbf{B} / B \omega_{c}\right) \times\left(v_{\|}^{2} \boldsymbol{\kappa}+\mu \nabla B\right)$ is the magnetic drift velocity, and $\boldsymbol{\kappa}$ is the magnetic field curvature. Note that the vector potential, defined by $\mathbf{A}=\mathbf{A}_{\|}-i A_{\perp} \mathbf{B} \times \mathbf{k}_{\perp} /\left(B k_{\perp}\right)$, is related to $\Phi, \Psi$ and $\delta B_{\|}$by $\omega \mathbf{A}_{\|}=-i \nabla_{\|}(\Phi-\Psi)$ and $\delta B_{\|}=k_{\perp} A_{\perp}$. We also note that the gyrokinetic formulation is still valid for the case $\rho_{i} \sim L_{\perp}$ if the the magnetic drift frequency is replaced by the pitch angle average value 
to account for the non-conservation of the magnetic moment [Hurricane et al.(1994)].

For electrons we shall neglect gyro-radius effects and consider $\left|v_{\|} \nabla_{\|}\right| \gg \omega, \omega_{d e}$. Clearly, trapped and un-trapped electrons have very different parallel dynamics. The un-trapped electron dynamics is mainly determined by its fast parallel transit motion, and to the lowest order in $\left(\omega /\left|v_{\|} \nabla_{\|}\right|\right)$the perturbed un-trapped electron density is given by [Cheng(1982a)]

$$
\delta n_{e u}=\frac{e N_{e u}}{T_{e}}\left[\frac{\omega_{* e}}{\omega} \Phi+\left(1-\frac{\omega_{* e}}{\omega}\right) \Psi\right],
$$

where $\omega_{* e}=\mathbf{B} \times \nabla N_{e} \cdot \mathbf{k}_{\perp} T_{e} /\left(B m_{e} \omega_{c e} N_{e}\right)$ is the electron diamagnetic drift frequency, $N_{e u} / N_{e}=1-\left[1-B(s) / B_{i}\right]^{1 / 2}$ is the fraction of un-trapped electron at the field line location $s, B_{i}$ is the maximum magnetic field along a field line. Near the minimum B location $N_{e u} / N_{e} \simeq B(s) / 2 B_{i} \ll 1$. Note that the first term in Eq. (2) is the adiabatic response and the second term is due to the parallel electric field.

The trapped electron dynamics is mainly determined by its fast parallel bounce motion and to the lowest order in $\left(\omega / \omega_{b e}\right)$

$$
\delta n_{e t} \simeq \frac{e N_{e t}}{T_{e}}\left[\frac{\omega_{* e}}{\omega} \Phi+\left(1-\frac{\omega_{* e}}{\omega}\right) \Delta \Psi\right]+\delta \hat{n}_{e t},
$$

where $N_{e t} / N_{e}=\left[1-B(s) / B_{i}\right]^{1 / 2}$ is the fraction of trapped electron,

$$
\begin{gathered}
\Delta=\int_{t r} d^{3} v\left(F_{e} / N_{e t}\right)\left[1-\frac{\left\langle\left(\omega-\omega_{d e}\right) \Psi\right\rangle}{\left\langle\omega-\omega_{d e}\right\rangle \Psi}\right], \\
\delta \hat{n}_{e t}=-\int_{t r} d^{3} v\left(e F_{e} / T_{e}\right)\left[\left(\omega-\omega_{\star e}^{T}\right) /\left(\omega-\left\langle\omega_{d e}\right\rangle\right)\right]\left\langle\left(\omega_{d e} / \omega\right) \Phi+v_{\perp}^{2} \delta B_{\|} / 2 \omega_{c e}\right\rangle,
\end{gathered}
$$

and $\left\langle\omega_{d e}\right\rangle$ is the trapped particle orbit average of $\omega_{d e}$. Note that the contribution due to the parallel electric field is small because $\Delta \ll 1$ for trapped electrons. Thus, it is difficult to change the trapped electron density by the parallel electric field because of their fast bounce motion relative to the parallel wave motion.

To obtain perturbed ion distribution function we assume that $\omega, \omega_{d i} \gg\left|v_{\|} \nabla_{\|}\right|$, and the non-adiabatic perturbed distribution function is given by

$$
g_{i} \simeq \frac{e F_{i}}{T_{i}} \frac{\omega-\omega_{\star i}^{T}}{\omega-\omega_{d i}}\left(\frac{\omega_{d i} J_{0} \Phi}{\omega}+\frac{v_{\perp} J_{1} \delta B_{\|}}{k_{\perp}}\right) .
$$

Note that the ion dynamics is mainly determined by its perpendicular motion and the perturbed ion density is given by

$$
\delta n_{i}=-\frac{e N_{i}}{T_{i}}\left[\frac{\omega_{* i}}{\omega} \Phi+\left(1-\frac{\omega_{* p i}}{\omega}\right)(1-\Gamma) \Phi\right]+\delta \hat{n}_{i},
$$


where $\omega_{* i}=\mathbf{B} \times \nabla N_{i} \cdot \mathbf{k}_{\perp} T_{i} /\left(B m_{i} \omega_{c i} N_{i}\right), \omega_{* p i}=\mathbf{B} \times \nabla P_{i} \cdot \mathbf{k}_{\perp} T_{i} /\left(B m_{i} \omega_{c i} P_{i}\right), \Gamma\left(b_{i}\right)=$ $I_{0}\left(b_{i}\right) \exp \left(-b_{i}\right), b_{i}=k_{\perp}^{2} T_{i} / M_{i} \omega_{c i}^{2}=k_{\perp}^{2} \rho_{i}^{2} / 2, I_{0}$ is the modified Bessel function of the zeroth order, and $\delta \hat{n}_{i}=\int d^{3} v g_{i} J_{0}$.

¿From the charge quasi-neutrality condition we obtain the parallel electric field potential

$$
\begin{array}{r}
\left(\frac{N_{e u}+N_{e t} \Delta}{N_{e}}\right) \Psi=-\frac{T_{e}}{T_{i}} \frac{\omega-\omega_{* p i}}{\omega-\omega_{* e}}(1-\Gamma) \Phi \\
+\frac{T_{e}}{e N_{e}}\left(\delta \hat{n}_{i}-\delta \hat{n}_{e}\right)
\end{array}
$$

In comparison with the limit without trapped electron effects, the parallel electric field is enhanced by $N_{e} /\left(N_{e u}+N_{e t} \Delta\right)$. Making use of the parallel Ampere's law the perturbed parallel current is given by $\delta J_{\|} \simeq i \nabla_{\perp}^{2} \nabla_{\|}(\Phi-\Psi) / \omega$, which represents the enhancement of stabilizing field line tension due to the enhanced parallel electric field resulting from effects of trapped electron dynamics and ion FLR.

To obtain the eigenmode equation for ballooning instability we follow the derivation presented in the paper by [Cheng et al.(1995)]. By multiplying the gyrokinetic equation with particle charge, integrating it over the velocity space and summing it over all species, and making use of the parallel component of the Ampere's law we obtain

$$
\begin{gathered}
\mathbf{B} \cdot \nabla\left[\frac{k_{\perp}^{2}}{B^{2}} \mathbf{B} \cdot \nabla(\Phi-\Psi)\right]+\frac{\omega\left(\omega-\omega_{* p i}\right)}{V_{A}^{2}} \frac{1-\Gamma\left(b_{i}\right)}{\rho_{i}^{2} / 2} \Phi \\
+\frac{\mathbf{B} \times \boldsymbol{\kappa} \cdot \mathbf{k}_{\perp}}{B^{2}}\left(\frac{2 \mathbf{B} \times \nabla P \cdot \mathbf{k}_{\perp}}{B^{2}} \Phi-\omega \sum_{j} \delta \hat{p}_{j}\right)=0
\end{gathered}
$$

where $V_{A}=B /\left(n_{i} M_{i}\right)^{1 / 2}$ is the Alfvén speed, and the non-adiabatic perturbed pressures for each particle species are given by $\delta \hat{p}_{j}=M_{j} \int d^{3} v\left[\left(1-\omega_{\star j}^{T} / \omega\right)\left(1-J_{0}^{2}\right) \Phi+g_{j} J_{0}\right]\left(v_{\perp}^{2} / 2+\right.$

$\left.v_{\|}^{2}\right)$. Note that the perturbed total pressure balance relation, $\mathbf{B} \cdot \delta \mathbf{B}+\delta P_{\perp} \simeq 0$, is used for low frequency instabilities with $\omega \ll k_{\perp} V_{A}$ [Cheng(1991), Cheng and Qian(1994), Cheng et al.(1995)].

Equations (8) and (9) form a coupled set of kinetic ballooning eigenmode equations for solving $\Phi$ and $\Psi$ along the field lines and the eigenvalue $\omega$. We also need to obtain the non-adiabatic contributions of perturbed electron density, $\delta \hat{n}_{e t}$ and $\delta \hat{n}_{i}$, and perturbed particle pressures, $\delta \hat{p}_{\|}$and $\delta \hat{p}_{\perp}$. The eigenmode equations include kinetic effects of trapped electron dynamics, parallel electric field, full ion FLR, and wave-particle resonances.

\section{$3 \quad$ Kinetic Ballooning Instability}

If we further consider the ordering $\omega \gg \omega_{d e}, \omega_{d i}$, the non-adiabatic density and pressure responses in Eqs. (8) and (9) can be neglected and we obtain a kinetic ballooning mode 
equation that retains the trapped electron and ion FLR effects, and the local dispersion relation for $\mathrm{KBI}$ is approximately given by

$$
\frac{\omega\left(\omega-\omega_{* p i}\right)}{\left(1+b_{i}\right) V_{A}^{2}} \simeq S k_{\|}^{2}-\frac{2 \kappa \cdot \nabla P}{B^{2}},
$$

where $S=1+\left(b_{i} /\left(1+b_{i}\right)\right) N_{e} T_{e} /\left(N_{e u}+N_{e t} \Delta\right) T_{i} \gg 1$, and we have adopted the Padé approximation $1-\Gamma \simeq b_{i} /\left(1+b_{i}\right)$. The real frequency of $\mathrm{KBI}$ is $\omega_{r} \simeq \omega_{* p i} / 2$ and the critical $\beta$ is given by

$$
\beta_{c} \simeq S \beta_{c}^{M H D}+\frac{\omega_{* p i}^{2} R_{c} L_{p}}{4\left(1+b_{i}\right) V_{A}^{2}},
$$

where $R_{c}$ is the radius of the magnetic field curvature and $L_{p}$ is the pressure gradient scale length, and $\beta_{c}^{M H D}=k_{\|}^{2} R_{c} L_{p}$ is the ballooning instability threshold based on the MHD theory.

We now briefly summarize the physical processes of kinetic stabilization effects of trapped electron dynamics and finite ion Larmor radii. In the substorm late growth phase the temperatures of electrons and ions are of the same order and the electron thermal velocity to be much larger than the ion thermal velocity. Because the frequency of kinetic ballooning instability is on the order of the ion diamagnetic drift frequency, its wave phase speed along the field line is usually much smaller than the electron thermal speed, but much larger than the ion thermal speed. Therefore, with respect to the parallel wave motion electrons move very rapidly along the field line with either transit or bounce motion depending on the particle pitch angle. On the other hand, ions moves very slowly with respect to the parallel wave motion and their parallel dynamics can be considered as static. Moreover, electron and ion motions across magnetic field lines are very different if the perpendicular wavelength is on the order of ion gyroradii; the electron perpendicular motion is essentially the $\mathbf{E} \times \mathbf{B}$ drift motion because of small mass, but the ion perpendicular motion is governed by both the $\mathbf{E} \times \mathbf{B}$ and polarization drifts. The difference in electron and ion motion across magnetic field lines can cause significant charge separation. In order to maintain the charge quasi-neutrality a parallel electric field must be produced to accelerate (or decelerate) electrons to positions where there is excess positive charge. A parallel electric field can easily accelerate (or decelerate) un-trapped electrons to change its density distribution. However, it is relatively harder to change the trapped electron density distribution by a parallel electric field because of their rapid bounce motion along the field lines. Thus, if the trapped electron population is larger than the un-trapped electron population, an enhanced parallel electric field will be produced to move electrons to maintain charge quasi-neutrality. The large parallel electric field will then drive an enhanced parallel current which can greatly increase the stabilizing field line tension over the value expected from the MHD theory 
just like high-pressured water increases the tension of a hose. As a result, a much higher $\beta_{c}$ than that based on the ideal MHD model is obtained with $\beta_{c} \approx O\left(N_{e} / N_{e u}\right) \beta_{c}^{M H D}$, where $N_{e u} / N_{e}<1$ is the un-trapped electron fraction and $\beta_{c}^{M H D}$ is the critical $\beta$ predicted by the ideal MHD theory. Therefore, for systems with a large fraction of trapped electron population the ideal MHD ballooning mode theory underestimates the critical $\beta$ for instability and it is necessary to use the more complete kinetic theory.

The kinetic ballooning instability can interact with perpendicular ion motion via wave-ion magnetic drift resonance because ion moves much slower than the wave along the field lines. If $\omega \sim \omega_{d i}$, the wave-ion magnetic drift resonance can provide an additional channel to release the KBI free energy and the growth rate and critical $\beta$ can be modified. To fully evaluate the effect of wave-ion magnetic drift resonance, we need to retain ion non-adiabatic responses in perturbed density and pressures. Numerical studies of KBI have been performed for tokamaks previously [Cheng(1982b), Cheng(1982a)] and the results indicated that the effect of the wave-ion magnetic drift resonance is to reduce $\beta_{c}$ by about $20 \%$ and the real frequency of KBI will increase to $\omega_{* p i}$ at critical $\beta$. We expect the results for the magnetosphere to be qualitatively similar to the tokamak case and the detailed numerical solutions will be presented in the future.

Another consequence of the wave-particle resonance is to produce a perturbed resonant ion distribution in the velocity space centered around $v_{y}=v_{d i}$ that oscillates with $\mathrm{KBI}$, where $v_{y}$ is the particle velocity in the duskward direction and $v_{d i}$ is the resonant ion magnetic ( $\nabla B$ and curvature) drift velocity which is approximately equal to the average drift velocity. As KBI grows to a large amplitude, the perturbed ion velocity distribution enhances the duskward ion flux in one half of the wave phase and vice versa for the other half of the wave phase. The resulting change in ion velocity distribution gives rise to $\partial f_{i} / \partial v_{y}>0$ near $v_{y} \sim v_{d i}$, and provides an additional free energy source for exciting higher frequency instabilities.

One consequence of the wave-ion magnetic drift resonance is that as KBI grows to a large value with $\delta B / B \geq 0.3$ the perturbed resonant ion velocity distribution has a positive slope near the duskward resonant ion magnetic drift velocity. This can be clearly seen from the $\left(\omega-\omega_{d i}\right)$ resonance denominator in the perturbed ion distribution. Because $\omega_{r} \simeq \omega_{* p i} / 2$, the wave-ion magnetic drift resonance will occur at $v_{d i}=T_{i} \mathbf{B} \times \nabla P_{i} /\left(2 e P_{i} B^{2}\right) \simeq v_{t h i} \rho_{i} / 2 L_{p i}$, where $\rho_{i}$ is the ion Larmor radius and $L_{p i}$ is the ion pressure gradient scale length. Thus, $\left|v_{d i}\right| \sim v_{t h i}$ for $\rho_{i} \sim L_{p i}$.

\section{Magnetic Reconnection}

An alternative popular idea in the space plasma physics community regarding the mechanism responsible for the onset of substorm expansion phase is the magnetic reconnec- 
tion [Sweet(1958), Parker(1963), Petschek(1964)]. There are two elements in the magnetic reconnection process [Kulsrud(1998)]: one is the change of magnetic field topology, and the other is the rate of energy conversion from magnetic field energy to plasma thermal and flow energies. The study of the physical process of magnetic reconnection is still an intensive ongoing activity in the plasma physics community. Some critical issues of magnetic reconnection are still being pursued: how current sheets form and what the current sheet topology is; how magnetic field reconnects in a 3D sheared magnetic field; what causes reconnection (external force or internal waves or instabilities); what the reconnection rate is and is it steady or spontaneous; and how electrons and ions gain energy; etc.

Despite insufficient understanding of magnetic reconnection physics, there have been constant attempts to propose the magnetic reconnection as the substorm onset mechanism. In particular, the near-earth neutral line model [Nagai and Kamide(1995)] has gained popularity which postulates that the substorm expansion phase is caused by magnetic reconnection in the near-earth plasma sheet. The near-Earth neutral line model is based on the scenario that the plasma and magnetic field are pinched by oppositely directed flows from north and south to form a current sheet in the equatorial near-Earth plasma sheet region. As magnetic reconnection occurs via plasma dissipation, both Earthward and tailward flows are generated to transport plasma and magnetic flux. In order for magnetic reconnection to be a viable mechanism for the substorm onset, several serious difficulties of the near-Earth neutral line model associated with both observational and theoretical constraints must be addressed. In the following we discuss a few of these difficulties and how the kinetic ballooning instability theory can naturally explain some critical substorm observations.

First, the effort by MHD simulations to find out whether magnetic reconnection process can occur in the near-Earth plasma sheet region has been carried out. It is a common conclusion that under normal solar wind and IMF conditions observed during substorms magnetic reconnection does not occur in the near-earth plasma sheet region. Even when magnetic reconnection occurs in the near-Earth plasma sheet under extremely unrealistic solar wind and IMF conditions in the MHD simulations, the reconnection region is quite broad ( $>3$ hours in local time and $>3 \mathrm{R}_{\mathrm{E}}$ in radial direction) in the equatorial region [Ogino(1999)]. This is inconsistent with the observation that the substorm onset is initiated in a small localized region of less than $1 \mathrm{R}_{\mathrm{E}}$ in radius in the near-Earth plasma sheet at $x \simeq-10 \mathrm{R}_{\mathrm{E}}$ around midnight. This corresponds to a localized initial aurora brightening region of less than a few degrees wide in longitude and less than one degree wide in latitude in the ionosphere. On the other hand, it is natural for us to expect that the kinetic ballooning instability will be initiated in a localized region in the near-Earth plasma sheet where the the plasma $\beta$ is large, mag- 
netic curvature is strong and field line tension is weak so that the driving free energy is maximized.

Secondly, the AMPTE/CCE observation of magnetic field data during substorms has clearly identified a low frequency instability which is excited before substorm onset and continues to evolve and develop into a strong plasma turbulence during the expansion phase. There is no physical mechanism to explain the low frequency instability based on the near-Earth neutral line model. On the other hand, the low frequency instability can be best explained in terms of the kinetic ballooning instability which requires kinetic effects of finite ion Larmor radii and trapped electron dynamics to properly understand the high $\beta_{c}(\geq 50)$, the observed wave frequency and growth rate, and the associated enhanced parallel electric field that is required to accelerate particle into and out of the auroral ionosphere.

Thirdly, it is required that the magnetic reconnection process in the near-earth plasma sheet region must produce observable high speed Earthward plasma flows perpendicular to the field lines before and during the substorm expansion phase. It relies on the Earthward flow to carry plasma and magnetic flux to cause magnetic field dipolarization in the near-Earth plasma sheet region. The near-Earth neutral line model advocates have been arguing that the existence of high speed flows is manifested by the bursty bulk flows (BBF). However, more recent analysis of the GEOTAIL data has indicated that there is no statistically favorable evidence of large perpendicular Earthward flows within $25 \mathrm{R}_{\mathrm{E}}$ from the Earth in the night side [Machida(1999)]. Moreover, recent detailed data analysis indicates that Earthward flows are either field-aligned ion beams or some types of MHD perturbations without significant $B_{z}$ flux.

\section{Summary}

In this paper we have identified a new scenario and physical processes of substorm explosive growth phase, onset and current disruption observed by AMPTE/CCE. We have found a low-frequency instability with a wave period of about 50-75 sec excited at approximately 2 minutes before the current disruption onset, and we have interpreted it as the kinetic ballooning instability (KBI). The $\beta$ threshold $(\geq 50)$ for exciting KBI is at least $10^{2}$ larger than that based on the ideal MHD theory because of the kinetic effects of trapped electron dynamics and finite ion Larmor radii which give rise to a large parallel electric field and hence a parallel current that greatly enhances the stabilizing effect of field line tension. With the KBI theory we are able to explain the enhanced duskward ion flux which occurs only during the explosive growth phase $(\approx 30 \mathrm{sec})$ and can excite higher frequency instabilities such as the cross-field current instabilities (CCI) [Lui(1996)]. Thus, our new substorm scenario emphasizes KBI which 
can naturally account for the features of the explosive growth phase and the initiation of current disruption through a combination of KBI and higher frequency instabilities such as CCI. We have also discussed the difficulties of explaining the substorm onset by the magnetic reconnection process as proposed in the near-Earth neutral line model.

Finally, we point out that even though progress has been made in understanding the substorm onset mechanism from the model of kinetic ballooning instability, more works are still needed to carry out a conclusive demonstration of substorm process by both theory and observation.

\section{Acknowledgments}

The author would like to thank Profs. T. Ogino and Y. Kamide of Solar-Terrestrial Environment Laboratory, Nagoya University, Japan for useful discussion. This work is supported by the NSF Grants No. ATM-9523331 and the DoE Contract No. DEAC02-76-CHO3073.

\section{References}

[Cheng(1982a)] Cheng, C. Z., High-n collisionless ballooning modes in axisymmetric toroidal plasmas, Nucl. Fusion, 22, 773, 1982a.

[Cheng(1982b)] Cheng, C. Z., Kinetic theory of collisionless ballooning modes, Phys. Fluids, 25, 1020, 1982b.

[Cheng(1991)] Cheng, C. Z., A kinetic-magnetohydrodynamic model for low-frequency phenomena, J. Geophys. Res., 96, 21159, 1991.

[Cheng and Qian(1994)] Cheng, C. Z., and Q. Qian, Theory of ballooning-mirror instabilities for anisotropic pressure plasmas in the magnetosphere, J. Geophys. Res., 99, 11193, 1994.

[Cheng et al.(1995)] Cheng, C. Z., N. N. Gorelenkov, and C. T. Hsu, Fast particle destabilization of TAE modes, Nucl. Fusion, 35, 1639, 1995.

[Cheng and Lui(1998)] Cheng, C. Z., and A. T. Y. Lui, Kinetic Ballooning Instability for Substorm Onset and Current Disruption Observed by AMPTE/CCE, Geophys. Res. Lett., 25, 4091, 1998. 
[Hurricane et al.(1994)] Hurricane, O. A., R. Pellat, and F. V. Coroniti, The kinetic response of a stochastic plasma to low frequency perturbations, Geophys. Res. Lett., 21, 253, 1994.

[Liu(1997)] Liu, W. W., Physics of the explosive growth phase: Ballooning instabilities revisited, J. Geophys. Res., 102, 4927, 1997.

[Kulsrud(1998)] Kulsrud, R. M., Magnetic reconnection in a magnetohydromagnetic plasma, Phys. Plasmas, 5, 1599, 1998.

[Lui(1996)] Lui, A. T. Y., Current disruption in the Earth's magnetosphere: Observations and models, J. Geophys. Res., 101, 13067, 1996.

[Lui et al.(1992)] Lui, A. T. Y., et al., Current disruptions in the near-Earth neutral sheet region, J. Geophys. Res., 97, 1461, 1992.

[Machida(1999)] Machida, S., private communication, 1999.

[Nagai and Kamide(1995)] Nagai, T., and Y. Kamide,, Magnetic field changes at the neutral sheet associated with substorm expansion onset: A model prediction and observation, J. Geophys. Res., 100, 3521, 1995.

[Ogino(1999)] Ogino, T., private communication, 1999.

[Ohtani et al.(1992)] Ohtani, S., K. Takahashi, L. Zanetti, T. A. Potemra, R. W. McEntire, and T. Iijima, Initial signatures of magnetic field and energetic particle fluxes at tail reconfiguration: Explosive growth phase, J. Geophys. Res., 97, 19311, 1992.

[Ohtani et al.(1995)] Ohtani, S., T. Higuchi, A. T. Y. Lui, and K. Takahashi, Magnetic fluctuations associated with tail current disruption: Fractal analysis, J. Geophys. Res., 100, 19135, 1995.

[Parker(1963)] Parker, E. N., The solar flare phenomenon and the theory of reconnection and annihilation ofmagnetic fields, Astrophys. J. Suppl. Ser., 8, 177, 1963.

[Petschek(1964)] Petschek, H. E., Magnetic field annihilation, in AAS-ASA Symposium on the Physics of Solar Flare, ed. by W. N. Hess, NASA SP-50, Washington, DC, 425, 1964.

[Roux et al.(1991)] Roux, A., S. Perraut, A. Morane, P. Robert, A. Korth, G. .Kremser, A. Pederson, R. Pellinen, and Z. Y. Pu, Plasma sheet instability related to the westward traveling surge, J. Geophys. Res., 96, 17697, 1991. 
[Sweet(1958)] Sweet, P. A., The neutral point theories of solar flares, in Electromagnetic Phenomena in Cosmic Physics, ed. E. Lehnert, 135, 1958.

[Voronkov et al.(1997)] Voronkov, L., R. Rankin, P. Frycz, V. T. Tikonchuk, and J. C. Samson, Coupling of shear flow and pressure gradient instabilities, J. Geophys. Res., 102, 9639, 1997. 